\title{
NONEXISTENCE OF INVARIANT MEASURES
}

\author{
DAVID PROMISLOW
}

\begin{abstract}
Let $G$ be a group acting on a set $X$. Suppose that for some positive integer $r, G$ contains a free group $F$ of rank $>r$ and the intersection of any stabilizer with $F$ has rank $\leqslant r$. A graph theoretic approach is used to show that there is no invariant measure on $X$.
\end{abstract}

1. Introduction. Let $G$ be a group acting on a set $X$. By an invariant measure for this action we will mean a finitely additive, nonnegative measure $\mu$, defined on all subsets of $X$, such that $\mu(X)=1$ and $\mu(g A)=\mu(A)$, for all $g \in G$ and $A \subseteq X$. For any $x \in X$ let $G_{x}$ denote the stabilizer subgroup at $x$. That is,

$$
G_{x}=\{g \in G: g x=x\} .
$$

Consider the question of whether or not an invariant measure exists. If $G$ is amenable (i.e. an invariant measure exists when $X=G$ and the action is by multiplication), it is not difficult to show that an invariant measure exists for all actions (see Greenleaf [5]). Our question then is of interest only for nonamenable groups. In particular we consider groups which contain a nonabelian free group. These are always nonamenable, and until recently were the only known examples of such.

In [10, Proposition 3.5] Rosenblatt shows that if an invariant measure exists, and $G_{x}$ is amenable for all $x$, then $G$ is amenable. It follows immediately that if $G$ contains a free group $F$ of rank greater than 1 and if for all $x, G_{x} \cap F$ is of rank $\leqslant 1$, therefore abelian, then no invariant measure exists. This same result was obtained independently by Akemann, using quite different methods (see [1, Proposition 4.7]). The purpose of this note is to prove the following generalization of the above result.

THEOREM. Let $G$ act on $X$. Suppose that for some positive integer $r, G$ contains a free group $F$ with rank $\geqslant r+1$ (possibly infinite) and that $G_{x} \cap F$ has rank $\leqslant r$ for all $x \in X$. Then no invariant measure exists.

Our techniques seem to differ somewhat from the usual ones used on problems of this type. We use a graph theoretic approach. Before giving the proof we will review briefly some background concepts.

2. Graph theory concepts. We will basically follow the terminology of Imrich [7]. See also Berge [2, Chapter 2]. Let $\Gamma=(V, E)$ be a connected directed graph where $V$ is the set of vertices and $E$ the set of edges. To each $e \in E$ we associate the inverse

Received by the editors July 6, 1982.

1980 Mathematics Subject Classification. Primary 43A07. 
edge $e^{-1}$ for which we reverse the initial and terminal vertices of $e$. The inverse edges are considered distinct from the set $E$. Fix a vertex $p$. Let $C_{p}$ be the set of all walks (i.e. a finite sequence of adjacent edges or inverse edges) beginning and ending at $p$. Under a natural equivalence relation and operation, the equivalence classes of $C_{p}$ form a group $\pi_{1}(p)$, called the fundamental group of the graph at $p$. This turns out to be a free group and its rank $\nu(\Gamma)$, independent of $p$ by connectivity, is called the cyclomatic number of the graph $\Gamma$.

For another definition of cyclomatic number, consider the map $T$ from $C_{p}$ into the vector space of all real valued functions on $E$, defined as follows. For any $\omega \in C_{p}$ and $e \in E$, let

$$
\begin{aligned}
{[T(\omega)](e)=} & (\text { number of occurrences in } \omega \text { of } e) \\
& -\left(\text { number of occurrences in } \omega \text { of } e^{-1}\right) .
\end{aligned}
$$

The subspace $Z$ generated by the image of $T$, which is independent of $p$ by connectivity, is called the cycle space of $\Gamma$ and its dimension is $\nu(\Gamma)$. From this formulation it is immediate that for a connected subgraph $\Gamma^{\prime}=\left(V^{\prime}, E^{\prime}\right)$.

$$
\nu\left(\Gamma^{\prime}\right) \leqslant \nu(\Gamma)
$$

since the cycle space of the subgraph can be identified with a subspace of $Z$, namely those functions in $Z$ which vanish on the edges not in $E^{\prime}$.

Let || denote cardinality. It is well known that when $V$ and $E$ are finite

$$
\nu(\Gamma)=|E|-|V|+1
$$

To any action of a group $G$ on $X$ and a subset $S$ of $G$ we associate a graph $\Gamma$ as follows. The vertex set is $X$ and the edge set is $(S \times X)$. The edge $(g, x)$ has initial vertex $x$ and terminal vertex $g x$. (This is known as the Caley graph in the case that $X=G$ and the action is by multiplication.) Let $F$ be the group generated by $S$. Then the orbits for the restriction of the action to $F$ correspond to the connected components of $\Gamma$. Fix a vertex $p$ and let $\Gamma_{p}$ be the component containing $p$. The map which assigns $g$ to the edge $e=(g, x)$, and $g^{-1}$ to its inverse, induces a homomorphism from $\pi_{1}(p)$ onto $G_{p} \cap F$. This will be 1-1 precisely when $S$ forms a free set of generators for $F$. So in such a case we have

$$
\operatorname{rank}\left(G_{p} \cap F\right)=\nu\left(\Gamma_{p}\right) .
$$

\section{Conclusion.}

Proof of The Theorem. We first reduce to the case where $F$ is finitely generated. Suppose that $A$ is an infinite set of free generators for $F$. Choose any finite $A_{0} \subseteq A$ of cardinality $>r$ and let $F_{0}$ be the subgroup generated by $A_{0}$. Using, for example, the Kurosch subgroup theorem [6, Theorem 17.3.1] or [8, p. 117, Exercise 32], we see that for any subgroup $H$ of $F, H \cap F_{0}$ is a factor in some free product decomposition of $H$ and so $\operatorname{rank}\left(H \cap F_{0}\right) \leqslant \operatorname{rank}(H)$. We can therefore replace $F$ by the finitely generated group $F_{0}$. Accordingly let $S=\left\{g_{1}, g_{2}, \ldots, g_{t}\right\}$ be a set of free generators for $F$ where $t$ is finite and $>r$. We first want to show that for any finite nonempty $Y \subseteq X$,

$$
\sum_{i=1}^{t}\left|g_{i} Y \cap Y\right| \leqslant(t-1)|Y|
$$


To do so we form the graph corresponding to $S$ as indicated above and let $\Gamma^{\prime}=\left(Y, E^{\prime}\right)$ be the full subgraph on $Y$ (i.e., $E^{\prime}$ consists of all edges with initial and terminal vertices in $Y$ ). We can assume that $\Gamma^{\prime}$ and $\Gamma$ are connected, since if (4) holds on each component, it will clearly hold globally. From (1), (2), (3) and our hypothesis on rank,

$$
\begin{aligned}
\left|E^{\prime}\right| & =\nu\left(\Gamma^{\prime}\right)-1+|Y| \leqslant \nu(\Gamma)-1+|Y| \leqslant(r-1)+|Y| \\
& \leqslant(r-1)|Y|+|Y|=r|Y| \leqslant(t-1)|Y| .
\end{aligned}
$$

This establishes (4), as the number of edges in $E^{\prime}$ with $g_{i}$ as first coordinate $=\left|Y \cap g_{i}^{-1} Y\right|=\left|g_{i} Y \cap Y\right|$ which shows that the left-hand side of (4) is just $\left|E^{\prime}\right|$.

We now appeal to the well-known Følner condition. See Rosenblatt [9] for a very general treatment. This condition says that an invariant measure exists iff given any finite nonempty set $S \subseteq G$ and any $\varepsilon>0$ there is a finite nonempty $Y \subseteq X$ such that

$$
|g Y \cap Y| \geqslant(1-\varepsilon)|Y|
$$

for all $g \in S$.

Given $S$ as above, $\varepsilon<t^{-1}$, and any finite nonempty $Y$, (6) cannot hold for all $g \in S$ as this would contradict (4). Hence, no invariant measure exists.

Remark. The theorem is obviously false for $r=0$ since $F$ could be of rank 1 . An attempt to adapt the proof would break down precisely on the last line of (5), since $(r-1)<0$.

EXAMPLE. In the case that $F$ has infinite rank the theorem is not necessarily true if we simply require that each $G_{x} \cap F$ be of finite rank. The uniform bound is needed. Consider the following example, which appeared in [3]. $G$ is generated by $\left\{g_{1}, g_{2}, g_{3}, \ldots, h_{1}, h_{2}, h_{3}, \ldots\right\}$ subject to the relations that $h_{i}$ and $h_{j}$ commute for all $i, j$ and that $g_{i}$ and $h_{j}$ commute for $i \leqslant j$. Let $F$ be the subgroup generated by $\left\{g_{1}, g_{2}, \ldots\right\}$, a free group of infinite rank. Let $X$ consist of all nonidentity elements of $G$ and let $G$ act on $X$ by conjugation. For $x$ equal to a product of $h_{i}$ 's and their inverses, with $s$ being the minimum index $i$ which is needed, $G_{x} \cap F=$ the group generated by $\left\{g_{1}, g_{2}, \ldots, g_{s}\right\}$. For all other $x, G_{x} \cap F$ is trivial. There is however an invariant measure. In other words, $G$ is inner amenable in the terminology of [4]. This follows from the results of [ 3 and 4] but the most direct way to see this is simply to use the Følner condition. For any finite subset $S$ of $G$ there is a one point $S$-invariant set, namely $\left\{h_{N}\right\}$ for $N$ sufficiently large. It does follow from our theorem that the measure of a finite union of conjugacy classes will be zero for any invariant measure on $X$.

ACKNowledgement. I would like to thank R. G. Burns for some helpful discussions.

\section{REFERENCES}

1. C. A. Akemann, Operator algebras associated with Fuchsian groups, Houston J. Math. 7 (1981), 295-301.

2. C. Berge, Graphs and hypergraphs, North-Holland, Amsterdam, 1973.

3. J. Dixmier and E. C. Lance, Deux nouveaux facteurs de type $\mathrm{II}_{1}$, Invent. Math. 7 (1969), 226-234.

4. E. G. Effros, Property $\Gamma$ and inner amenability, Proc. Amer. Math. Soc. 47 (1975), 483-486. 
5. F. P. Greenleaf, Invariant means on topological groups and their applications, Van Nostrand Math. Studies, no. 16, New York, 1969.

6. M. Hall, Jr., The theory of groups, Macmillan, New York, 1959.

7. W. Imrich, Subgroup theorems and graphs, Combinatorial Mathematics V, Lecture Notes in Math., vol. 622, Springer-Verlag, Berlin and New York, 1977, pp. 1-27.

8. W. Magnus, A. Karrass and D. Solitar, Combinatorial group theory, Interscience, New York, 1966.

9. J. M. Rosenblatt, A generalization of Folner's condition, Math. Scand. 33 (1973), 153-170.

10. __ Uniqueness of invariant means, Trans. Amer. Math. Soc. 265 (1981), 623-636.

Department of Mathematics, York University, Downsview M3J 1P3, Ontario, Canada 